Yojcom, D. \& Ruiz, J. (2020). Los factores sociales asociados a los modelos matemáticos en un contexto de pandemia. Revista Latinoamericana de Etnomatemática, 13(1), 355-374. DOI: 10.22267/relatem.20131.47

Artículo recibido el 25 de julio de 2020; Aceptado para publicación el 10 de agosto de 2020

\title{
Los factores sociales asociados a los modelos matemáticos en un contexto de pandemia
}

\section{Social factors linked to mathematical models in a pandemic context}

\author{
Domingo Yojcom Rocché ${ }^{1}$ \\ Juan Carlos Ruiz Castillo ${ }^{2}$
}

\begin{abstract}
Resumen
La crisis provocada por la pandemia COVID-19 confirma la importancia de los conocimientos matemáticos que permite estimar comportamientos, a través de modelos para tener un panorama más amplio de lo que acontece. Los modelos matemáticos son utilizados en diferentes contextos especialmente: económicos, agrícolas, climáticos, financieros, tecnológicos, médicos, educativos, entre otros. Este artículo de tipo reflexivo toma elementos de la situación guatemalteca para presentar una aproximación de la salubridad que vive el país actualmente. El texto consta de tres partes: la primera hace una introducción histórica de la aplicación de modelos matemáticos, para disminuir el brote de contagios en epidemias o pandemias, con el fin de ayudar a la población a minimizar la propagación del virus. La segunda parte explica la construcción del modelo determinístico SIR con algunas adaptaciones para su interpretación, uno de los más utilizados en diversos países del mundo y la aplicación de estos conocimientos en contextos de diversidad cultural. En tanto, la tercera parte, enfatiza la importancia de los factores asociados al modelo para su implementación y funcionamiento en una sociedad multicultural. Dentro de los factores que aborda el artículo se encuentran: la capacidad de relacionar datos matemáticos, la vinculación de resultados al conocimiento unitario, la cultura como motor de desarrollo local, la naturaleza del fenómeno de estudio, la evolución como uno de los principios básicos de la vida, la educación y la capacidad de reacción para atender emergencias.
\end{abstract}

Palabras claves: Modelos Matemáticos; COVID-19; Factores asociados; Multiculturalidad; Conocimientos Ancestrales; Fenómenos Biológicos.

\begin{abstract}
The crisis caused by the pandemic COVID-19 confirms again the importance of mathematical knowledge to estimate natural phenomenon, actually various models been used to have a broader picture of what is happening. Mathematical models are used in different contexts especially: economic, agricultural, climatic, financial, technological, medical, educational, among others. This reflective article takes elements from the Guatemalan situation to present an approximation of the health that the country is currently experiencing. The text has of three parts: the first makes a historical introduction to the application of mathematical models to reduce the outbreak of contagion in epidemics or pandemics, in order to help the population, minimize the spread of the virus. The second part explains the construction of the SIR deterministic model with some adaptations for its interpretation, the most used in various countries of the world and the application of this knowledge in contexts of cultural diversity. Meanwhile, the third part emphasizes the importance of the factors associated with the model for its implementation and operation in a multicultural society. Among the factors addressed in the paper are: the ability to relate mathematical data, the relationship of results to unitary knowledge, culture as an engine of local development, the nature of the phenomenon of study, evolution as one of the basic principles of life, education and the ability to react to respond to emergencies.
\end{abstract}

\footnotetext{
${ }^{1}$ Doctor en Ciencias con especialidad en Matemática Educativa, Centro de Investigación Científica y Cultural, San Pedro La Laguna, Sololá, centrocientificogt@gmail.com

${ }^{2}$ Maestro en Ciencias y Licenciado en la Enseñanza de la Matemática y la Física, Universidad de San Carlos de Guatemala, Villa Nueva, Guatemala, 2jcruiz@gmail.com
} 
Keywords: Mathematical models; COVID-19; Associated factors; Multiculturality; Ancestral Wisdoms; Biological Phenomena.

\section{INTRODUCCIÓN}

Para la mayoría de las personas pareciera que la matemática jamás había estado tan presente en nuestro entorno como en este momento. La pandemia del coronavirus 2019 (COVID-19) ha despertado el interés de las personas en esta ciencia, por el uso de cifras numéricas e interpretación de gráficas. Las matemáticas son indispensables para los estudios científicos y tienen una gran influencia en las labores habituales, además, brindan herramientas para otras ciencias con el manejo de estimaciones y modelos que puedan ayudar a mejorar la comprensión de nuestro entorno.

En el caso del COVID-19, se han conseguido ver diferentes curvaturas, las cuales son dadas por medio de los modelos teóricos, entre ellos los más utilizados, son compartimentados y determinísticos. Los modelos matemáticos son entendidos en este texto como expresiones científicas empleadas para generalizar y estimar relaciones entre objetos y variables. Uno de los modelos determinísticos es el modelo SIR siglas que indican los grupos: $\mathrm{S}$ (susceptibles), I (infectados) y R (recuperados). Para implementar este modelo se divide la población en tres grupos: el grupo de los susceptibles, de los infectados y de los recuperados. Este modelo permite percibir, cómo los susceptibles se vuelven infectados por el parámetro $\alpha$ y los infectados se recuperan por el parámetro $\delta$.

Estos parámetros son números que identifican el comportamiento del brote, condicionado por factores sociales y culturales de la población que serán explicados posteriormente. Actualmente existen diversos artículos que tratan de hacer estimaciones de estos parámetros, empero por las pocas pruebas que se tienen en Guatemala; así como en otros países, es muy difícil evaluar el comportamiento de dichos fenómenos.

Según las cifras reveladas por el Secretario de Comunicación Social de la Presidencia de Guatemala, el país contaba con 22,500 pruebas que habían sido otorgadas por el Banco Centroamericano de Integración Económica (BCIE) el día 29 de marzo del presente año (Gramajo, 2020). Sin embargo Girón (2020) en la página la Hora; señala que desde el 13 de marzo al 13 de mayo del presente año, se contaba con 12,688 pruebas realizadas, a diferencia de otros países donde se aplican una cantidad muy grande de pruebas semanalmente, como 
lo señala la página France24: "Mientras Alemania hace un promedio de medio millón de test por semana, otros países europeos, como Italia o España, hacen una media que va entre los 50,000 y los 100,000 test por semana" (France24, 2020, párrafo 5) de allí emerge la siguiente pregunta ¿Cuáles son esos factores que condicionan estas cifras publicadas por los medios oficiales?

Es importante considerar que, a mayor número de pruebas, existe una mayor probabilidad de identificar los casos positivos. Sin embargo, con los datos que se tenían hasta esa fecha, se realizaron diferentes aproximaciones con un margen de error muy grande en comparación con los de otros países. Para adentrarnos un poco más a la comprensión del problema, es importante conocer algunos datos históricos del uso de la matemática, en algunas epidemias y/o pandemias.

\section{ANTECEDENTES DE LA MATEMÁTICA APLICADA ${ }^{3}$ EN EPIDEMIAS}

El desarrollo de modelos matemáticos de epidemias tuvo un gran progreso a principios del siglo XX. A causa de los contagios que azotaron a la población mundial, entre las cuales destaca la epidemia de la influenza del 1918 que mató a millones de personas (Galindo, Rodríguez y Cervantes (2014). En el siglo XVII, las matemáticas ayudaron a calcular la propagación de epidemias, como en el caso de Daniel Bernoulli, que formuló un modelo epidemiológico para la viruela, con el fin de combatir esta temida enfermedad. En Europa se planteó la posibilidad de adoptar la inoculación como medida preventiva. Esta práctica consistía en implantar una pequeña cantidad de material biológico, originario de una persona enferma a una sana, de modo que ésta desplegara una adaptación benigna de la enfermedad, con la expectativa que una vez superada la enfermedad, la persona estuviera inmune. Sin embargo, esta práctica se convirtió en un tema muy polémico, porque no estaba exenta de riesgos, ya que un pequeño porcentaje de los inoculados podía desarrollar la viruela y morir como consecuencia de esta (Herrero, 2017).

\footnotetext{
3 "el término matemáticas aplicadas se refiere a todos aquellos métodos y herramientas matemáticas que pueden ser utilizados en el análisis o solución de problemas pertenecientes al área de las ciencias aplicadas o sociales." (Ecured, 2020, p. 1).
} 
Es importante señalar que el modelo de Bernoulli, fue obtenido para describir la trasmisión de la enfermedad en una población. Dicho modelo relaciona el número de personas con edad $x$ de ser susceptibles a ser infectadas $(S(x))$, con el número de personas vivas con esa edad $(p(x))$, la relación esta enunciada con la siguiente expresión: $\frac{\mathrm{s}(\mathrm{x})}{\mathrm{p}(\mathrm{x})}=\frac{1}{(1-\mathrm{p}) \mathrm{e}^{\mathrm{qx}}+\mathrm{p}}$.

Para deducir la tasa de contagio $q$, Bernoulli conjeturó que el número de muertes por viruela figuraba 1/13 del total de muertes. Utilizando las tablas de Halley, concluyó que abarcaba atribuir a la viruela unas 100 del total de 1300 muertes registradas en dichas tablas.

Se comparó los valores proporcionados por la fórmula que había obtenido, con $\mathrm{p}=1 / 8$ y diversos valores de $q$, con los datos de personas vivas proporcionados por las mismas tablas, y dedujo así que el mejor ajuste correspondía a $\mathrm{q}=1 / 8$. Bernoulli atribuyó validez general a esos parámetros y consideró mera coincidencia el que sus valores fueran iguales (Herrero, 2017, p. 1).

Los valores no eran muy precisos porque fueron ignorados los factores asociados al fenómeno, pues como en todo cálculo matemático existe el cambio de variable, el cual presenta valores diferentes a lo estimado. Cabe mencionar que Bernoulli, con esta expresión pudo calcular el aumento en la esperanza de vida en caso de optar por contagio.

\subsection{MODELO SIR EN EL CASO DEL VIRUS, HEMAGLUTININA Y NEURAMIDASA (H1N1)}

En el 2009 surgió la epidemia $\mathrm{H} 1 N 1^{4}$ donde se utilizaron diversos modelos teóricos, que implicaron el uso de la dinámica de crecimiento del contagio, además su propagación mundial. En el estudio de Galindo, Rodríguez y Cervantes (2014) el modelo SIR fue utilizado para hacer el cálculo de la epidemia, el cual se representa así: $s \rightarrow I \rightarrow R$.

Este modelo presenta los siguientes postulados:

a) En la epidemia, una sola infección es la responsable de ocasionar un proceso infeccioso en el huésped.

b) El desenlace de la enfermedad es la muerte o la inmunidad completa.

c) La tasa de contagio $\mathrm{c}(\mathrm{t})$ es proporcional al número de enfermos, i.e., $\mathrm{c}(\mathrm{t})=\mathrm{aI}(\mathrm{t})$, siendo a $>0$ constante.

d) El número de individuos susceptibles que se contagian por unidad de tiempo es $\mathrm{c}(\mathrm{t}) \mathrm{S}(\mathrm{t})$; por lo tanto, la cantidad de personas que se contagian por unidad de tiempo es $a I(t) S(t)$ con $a>0$. Indicado de diferente modo la cantidad de susceptibles $s(t)$ al pasar un tiempo $\Delta t$ será $S(t+\Delta t)=S(t)-a I(t) S(t) \Delta t$.

e) Todos los individuos sanos son susceptibles.

\footnotetext{
${ }^{4}$ Es importante señalar que el modelo SIR fue creado por Kremarck y Mckendrick en 1927.
} 
f) La población $\mathrm{N}$ es cerrada para cualquier tiempo $S+I+R=N$. Es decir, no se toman en cuenta nacimientos ni migraciones.

g) El periodo de incubación que contempla el modelo más general de Kermack y McKendrick es muy corto. El individuo susceptible se enferma y puede contagiar a otro.

h) La velocidad de decremento de infecciosos es proporcional a su número. En términos matemáticos se representa así $b I(t) \operatorname{con} b>0$, donde $b$ es la razón de removidos por unidad de tiempo constante (Galindo, Rodríguez \& Cervantes, 2014, p. 239).

En el estudio se utilizaron otras expresiones para representar el número de infecciosos $I(t)$ luego de transcurrir un tiempo $\Delta t$ el cual se enuncia de la siguiente manera:

$I(t+\Delta t)=I(t)+a I(t) S(t) \Delta t-b I(t) \Delta t$

Los removidos $R(t)$ al transcurrir un tiempo $\Delta t$ será: $R(t+\Delta t)=R(t)+b I(t) \Delta t$

Para este modelo todas las personas que cohabitan en espacios geográficos, se interrelacionan e interactúan a excepción de los hospitalizados y fallecidos. Las matemáticas aportan aspectos para poder mejorar el estilo de vida, ante las amenazas que se presenten en diferentes contextos, pues no sólo dan la estimación en el brote de epidemias y/o pandemias; sino también ofrecen soluciones concretas para tomar decisiones.

\section{EL MODELO SIR EN LA PANDEMIA COVID-19}

Se tienen diferentes modelos teóricos para estimar las epidemias y/o pandemias entre las más conocidas tenemos: modelo SIR, SIR con nacimientos y muertes, SIRS, SEIS, SEIR, MSIER y el modelo MSERI ${ }^{5}$.

La ley de los grandes números señala que si se lleva a cabo repetidas veces un mismo experimento...la frecuencia con la que se repetirá un determinado suceso...se acercará a una constante. Dicha constante será a su vez la probabilidad de que ocurra este evento (Roldan, 2020, p.1).

Inicialmente, estos modelos pueden apreciar el contagio en los individuos, sin embargo, la ley de los grandes números afirma que la cantidad de infectados se van haciendo cada vez más previsible, conforme aumenta el tamaño de población. Estos modelos teóricos son utilizados para frecuentar padecimientos que afectan a poblaciones magnas, a menudo son representados por ecuaciones diferenciales.

\footnotetext{
${ }^{5}$ SIRS=susceptibles, infectados, recuperados y susceptibles; SEIS=susceptibles, expuestos, infectados y susceptibles; SEIR=susceptibles, expuestos, infectados y recuperados; MSIER= inmunes, susceptibles, infectados, expuestos y recuperados; MSERI= inmunes, susceptibles, expuestos, recuperados, infectados.
} 
Se puede considerar que, ante la diversidad de modelos, el modelo SIR puede adaptase al estudio epidemiológico, pues el que más características típicas se captura de los brotes epidémicos, siendo el más utilizado por la Organización Mundial de la Salud (OMS). El modelo SIR describe el comportamiento de tres grupos en los cuales están: susceptibles, infectados y recuperados.

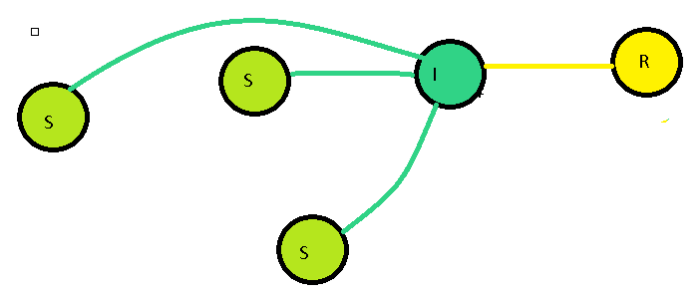

Figura 1. Relación de susceptibles (S), infectados (I) y recuperados (R). Fuente: Elaboración propia

En este modelo: $S$, son personas sin inmunidad al virus, y por consecuente están expuestas al mismo; $I$, personas infectadas que pueden ser trasmisoras del virus a las personas susceptibles; $R$, personas que se recuperaron y que no pueden afectar a otras con trasmitir el virus. Dados los términos S, I y R, se pueden establecer modelos para estas estimaciones por medio de sus parámetros. Se establece como $\alpha$ al parámetro de susceptibles a infectados y $\delta$ al parámetro de infectados a recuperados, ver figura 2.

Para Gerber (2012, 0:30 min.) "la probabilidad de contagio va a depender del tiempo del contagio, consecuentemente $\alpha d t$ es la probabilidad por unidad de tiempo de ser contagiado, en el caso $\delta d t$ es la probabilidad por unidad de tiempo de ser recuperado". Según este autor, el modelo SIR puede brindar estimaciones por medio de gráficas para encontrar soluciones que disminuyan la propagación del virus. El parámetro $\alpha$ obedece al número de personas con que el infectado se interrelaciona, por lo tanto: $\alpha d t C \frac{i}{n}$.

Donde $c$ es el número de personas con que se tiene contacto, $i$ es el número de personas infectadas y $n$ el número de personas totales, por lo tanto: $n=S+I+R$, el número susceptibles son los que tienen la probabilidad de ser contagiados. Sea $d s=-\alpha d t$ la probabilidad de contagiados, se puede señalar que es negativa por que se van a perder a 
personas que están susceptibles. Por lo tanto, el modelo de las personas susceptibles es el siguiente: $\frac{d S}{d t}=-\alpha c \frac{i}{n} s$.
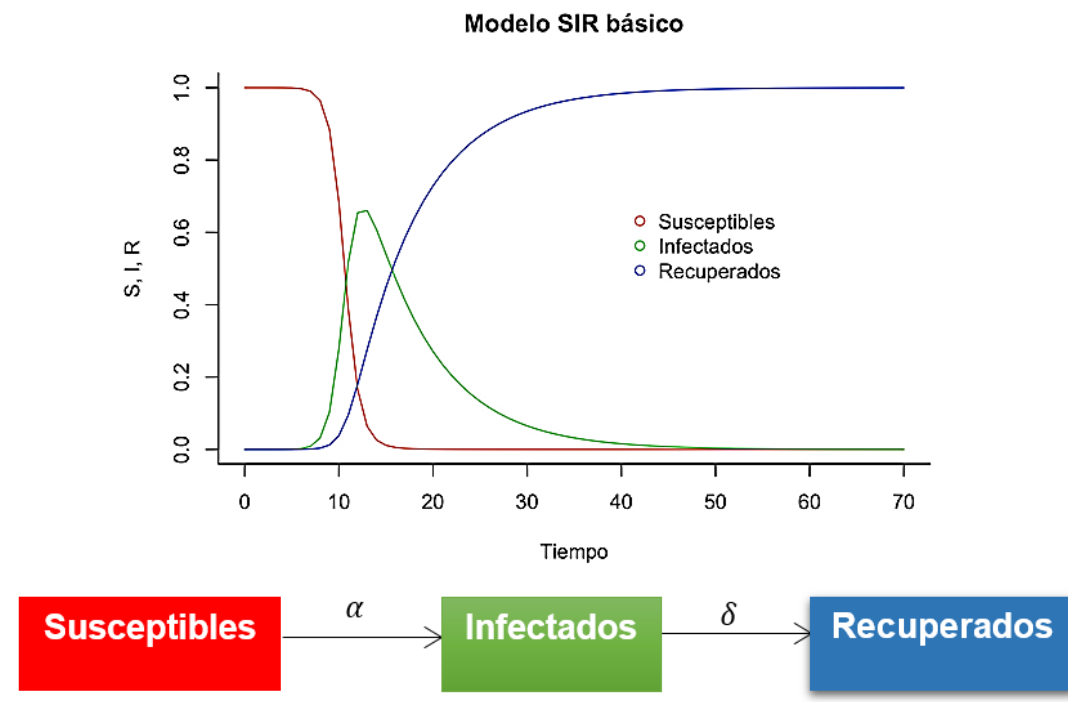

Figura 2. Modelo SIR básico.

Fuente. Adaptación de Fernández (2018).

Hamer, en el 1906, formuló la ley de masas, donde estableció que el número de contagiados de contactos infecciosos, es decir, que producen enfermedad, por unidad de tiempo es proporcional al número total de contactos entre individuos infecciosos y susceptibles (Cervantes, 2015, p. 171).

Continuando con el modelo de número de los infectados, se puede definir que es la magnitud de los susceptibles menos los recuperados con respecto al tiempo, como se ve en la siguiente función: $\frac{d I}{d t}=\alpha c \frac{i}{n} s-\frac{d r}{d t}$. Este es definido por el número de infectados con respecto al tiempo, siendo equivalente al parámetro $\alpha$ por el número de contactos multiplicados por la relación de infectados con el número de personas, multiplicados por los susceptibles menos el número de recuperados en un determinado tiempo (ver tabla 1).

\section{Tabla 1.}

\begin{tabular}{|l|c|}
\hline En el caso de los recuperados el modelo es & $\begin{array}{c}d r=\text { el número de recuperados } \\
d r=\delta i d t\end{array}$ \\
\hline Donde $\delta d t$ tes la probabilidad de recuperación & $\frac{d r}{d t}=\delta i$ \\
\hline
\end{tabular}




\begin{tabular}{|l|c|}
\hline $\begin{array}{l}i(t) \text { Es el total de personas infectadas en el tiempo, se } \\
\text { puede establecer qué y } I(t) \text { es el total de personas } \\
\text { infectas en el tiempo }\end{array}$ & $I(t)=\int_{0}^{t} d t^{\prime} i\left(t^{\prime}\right)-r(t)$ \\
\hline $\begin{array}{l}\text { La probabilidad de recuperación se expresa de la } \\
\text { siguiente manera }\end{array}$ & $R(t)=\int_{0}^{t} d t^{\prime} i\left(t^{\prime}\right) p\left(t-t^{\prime}\right)$ \\
\hline Reescribiendo esta ecuación & $t=\frac{1}{\alpha c} u$ \\
\hline $\begin{array}{l}\alpha c \text { es una escala de tiempo por la ecuación anterior. } \\
\text { Derivando y sustituyendo la ecuación obtendremos }\end{array}$ & $\frac{d S}{d u} \alpha c=-\alpha d t c \frac{I}{n} s$ \\
\hline Dividendo ambos lados por $c$ & $\frac{d S}{d u} \alpha=-\alpha d t \frac{I}{n} s$ \\
\hline Sustituyendo $d t$ como $c$ & $\frac{d S}{d u} \alpha=-\alpha c \frac{I}{n} s$ \\
\hline Haciendo la sustitución en el caso de los infectados & $\frac{d I}{d u} \alpha c=\alpha c \frac{I s}{n}-\delta I$ \\
\hline Dividiendo $\alpha c$ en ambos lados & $\frac{d I}{d u}=\frac{I s}{n}-\frac{\delta I}{\alpha c}$ \\
\hline Utilizando la propiedad distributiva obtendremos & $\frac{d I}{d u}=I\left(\frac{s}{n}-\frac{\delta}{\alpha c}\right)$ \\
\hline
\end{tabular}

Fuente: Elaboración propia.

Donde $s<n$ y $\delta<\alpha c$ en el caso de los infectados van a tener una pendiente positiva cuando $\frac{\delta}{\alpha c}<\frac{s}{n}$ y negativa $\frac{\delta}{\alpha c}>\frac{s}{n}$. El inverso de $\frac{\delta}{\alpha c}$ es conocido como factor de reproducción, el cual se define $\boldsymbol{R}_{\boldsymbol{o}}=\frac{\boldsymbol{\alpha} \boldsymbol{c}}{\boldsymbol{\delta}}$ este factor compara la velocidad con el crecimiento del virus y la velocidad de los que se están recuperando. Se puede establecer que: $R_{O}>1 \mathrm{y} \frac{d I}{d u}>0$ epidemia; $R_{o}<$ $1 \mathrm{y} \frac{d I}{d u}<0$ control.

Cada vez que la velocidad del crecimiento es más rápida que la velocidad con la que se están recuperando, el sistema es una epidemia y cada vez que la velocidad del crecimiento es menor de los que se está recuperando, se puede establecer el sistema está bajo el control. (ver tabla 2).

Tabla 2. 


\begin{tabular}{|c|c|}
\hline $\begin{array}{l}\text { Para poder hacer una gráfica con este modelo se } \\
\text { necesita relacionar los términos S, I, y R. Siendo }\end{array}$ & $\begin{array}{c}\frac{d S}{d u}=-\alpha c \frac{I}{n} s \\
\mathrm{y} \\
\frac{d I}{d u}=\alpha c \frac{I s}{n}-\delta I\end{array}$ \\
\hline $\begin{array}{l}\text { Estas expresiones permiten la creación de una } \\
\text { nueva ecuación al relacionarlas entre sí }\end{array}$ & $\frac{\frac{d I}{d u}}{\frac{d s}{d u}}=\frac{\alpha c \frac{I s}{n}-\delta I}{-\alpha c \frac{I s}{n}}$ \\
\hline Simplificando la expresión obtendremos & $\begin{array}{c}\frac{d I}{d S}=-1+\frac{n \delta}{\alpha c} \cdot \frac{1}{S} \\
d I=\left(-1+\frac{n \delta}{\alpha c} \cdot \frac{1}{S}\right) d S\end{array}$ \\
\hline Integrando ambos lados & $\begin{array}{c}\left(I-I_{o}\right) \rightarrow\left(S-S_{o}\right) \\
I-I_{o}=\left(-1+\frac{n \delta}{\alpha c} \cdot \frac{1}{S}\right)\left(S-S_{o}\right)\end{array}$ \\
\hline $\begin{array}{l}\text { Utilizando la propiedad distributiva } \quad \text { y } \\
\text { simplificando la expresión }\end{array}$ & $\begin{array}{c}I-I_{o}=-\left(S-S_{o}\right)+S \frac{n \delta}{\alpha c} \cdot \frac{1}{S}-S_{o} \frac{n \delta}{\alpha c} \cdot \frac{1}{S} \\
I-I_{o}=-\left(S-S_{o}\right)+\frac{n \delta}{\alpha c}+\int-S_{o} \frac{n \delta}{\alpha c} \cdot \frac{1}{s} \\
I-I_{o}=-\left(S-S_{o}\right)+\frac{n \delta}{\alpha c}+\int \frac{1}{s-s_{o}} \\
I-I_{o}=-\left(S-S_{o}\right)+\frac{n \delta}{\alpha c} \ln \frac{s}{s_{o}}\end{array}$ \\
\hline $\begin{array}{l}\text { Si suponemos que ya no se tienen infectados, ya } \\
\text { sea, porque se recuperaron o se quedaron en los } \\
\text { susceptibles, entonces, }\end{array}$ & $I=0, I_{o} \ll 0$ \\
\hline Sustituyéndolo en la ecuación & $-\left(S-S_{o}\right)+\frac{n \delta}{\alpha c} \ln \frac{s}{S_{o}}=0$ \\
\hline Despejando la ecuación & $\ln \frac{S}{S_{o}}=\frac{\alpha C}{n \delta}\left(S-S_{o}\right)$ \\
\hline
\end{tabular}

Fuente: Elaboración propia.

Se asumirá $s<s_{o}$, por lo tanto, el dato mayor que podrá obtener de esta relación es aproximadamente 1 (al asumir que los datos puedan ser iguales). Se hará la sustitución de la expresión $\frac{s}{s_{o}}=x$ (con fines que se pueda comprender otros aspectos), con lo cual obtendrá $\ln x$, al graficarlo se tendrá la figura 3 colocando la pendiente con el valor igual a 1. 


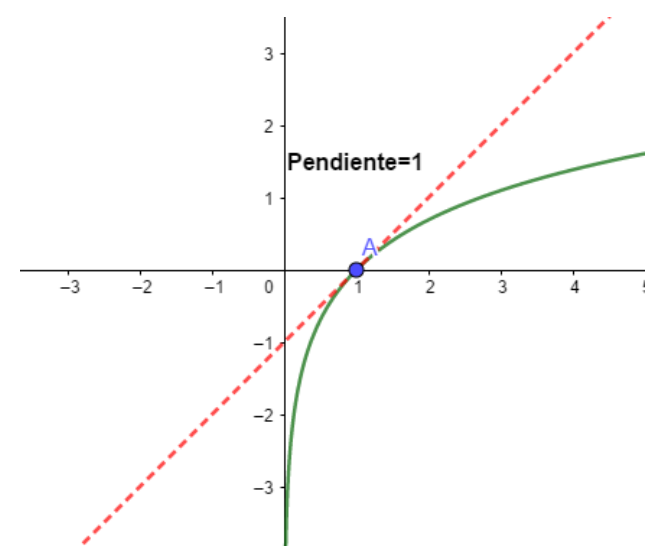

Figura 3. Gráfica de $\ln x$ con ecuación lineal.

Fuente: Elaboración propia con Geogebra.

Tabla 3.

\begin{tabular}{|l|c|}
\hline En el otro lado de la ecuación & $\frac{\alpha c}{n \delta}\left(S-S_{o}\right)$ \\
\hline $\begin{array}{l}\text { Utilizando la propiedad distributiva con } S_{o} \text { con lo que } \\
\text { obtenemos }\end{array}$ & $\frac{\alpha S_{o}}{n \delta}\left(\frac{S}{S_{o}}-1\right)$ \\
\hline Donde $\frac{\alpha S_{o}}{n \delta}$ es la pendiente, llegando a la siguiente expresión $m(x-1)$
\end{tabular}

Fuente: Elaboración propia.

Graficando la ecuación en la figura 4.

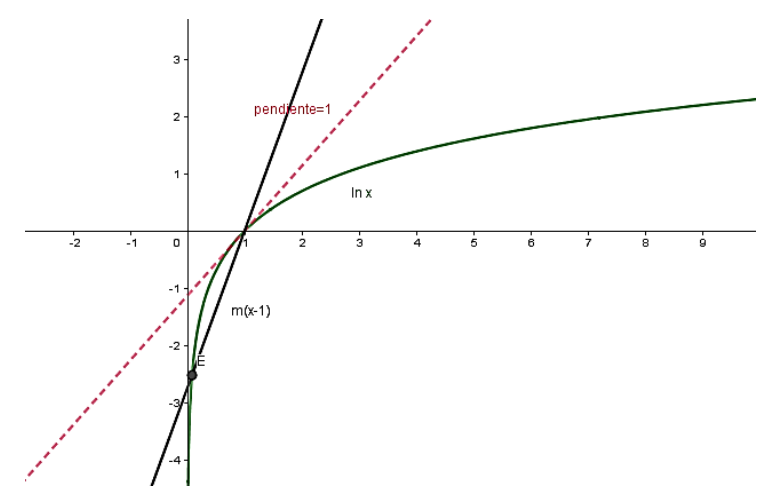

Figura 4. Relación de $\ln x \operatorname{con} m(x-1)$.

Fuente: Elaboración propia con Geogebra.

Se evidencia en el gráfico de la figura 4 que no intercepta la gráfica en un punto mayor a $x=$ 1 , porque no puede ser mayor según lo establecimos anteriormente.

Por lo tanto, $\frac{\alpha S_{o}}{n \delta}=m$. A continuación, en la figura 5 se muestra $m<1$ 


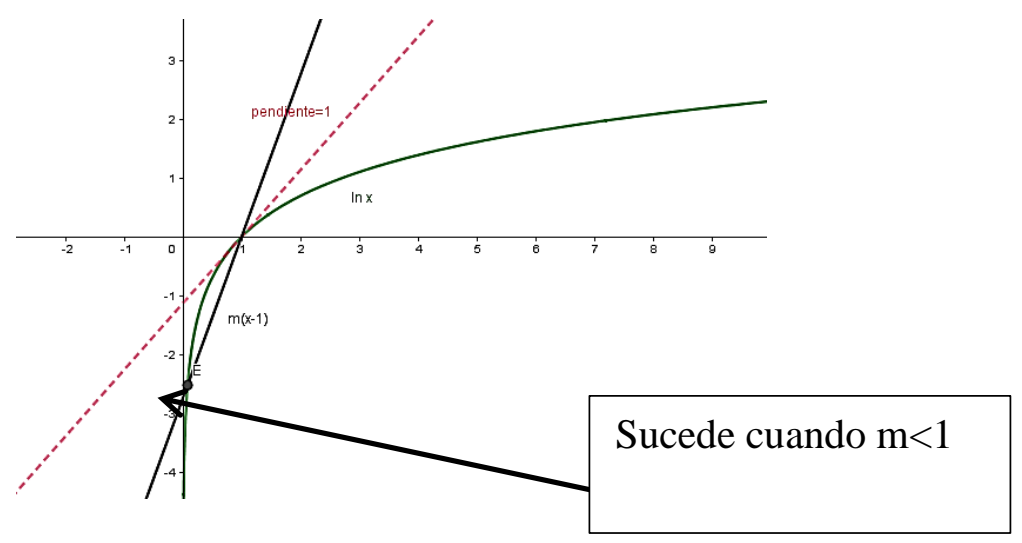

Figura 5. $m(x-1)$.

Fuente: Elaboración propia con Geogebra.

Esto señala que la solución se estabilizó, indicando que se tenía una pandemia, pero se pudo estabilizar en el caso que fuera $m<1$, representando de la siguiente manera en la figura 6 .

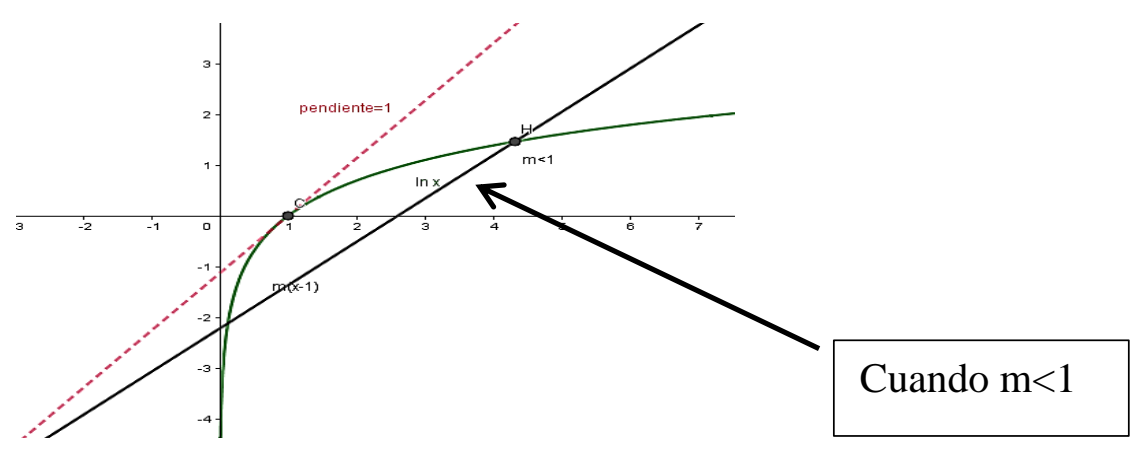

Figura 6. Representación de $m<1$.

Fuente: Elaboración propia con Geogebra

Indicando que no ha terminado el brote epidémico, como se observa en la tabla 4.

Tabla 4.

\begin{tabular}{|l|c|}
\hline Teniendo las sustituciones donde & $\frac{s}{s_{o}}=x \mathrm{y} \frac{\alpha S_{o}}{n \delta}\left(\frac{s}{s_{o}}-1\right)=m(x-1)$ \\
\hline podremos señalar que ln $x$ es entorno a & $1 \sim(x-1)-\frac{(x-1)^{2}}{2} \cdots$ \\
Sustituyendo en la ecuación & $(x-1)-\frac{(x-1)^{2}}{2}=m(x-1)$ \\
\hline Dividiendo $(x-1)$ en la ecuación obtendremos & $1-\frac{(x-1)}{2}=m$ \\
\hline $\begin{array}{l}\text { Multiplicando por } 2 \\
\text { Por propiedad distributiva }\end{array}$ & $2-(x-1)=2 m$ \\
\hline Despejando $x$ & $2-x+1=2 m$ \\
\hline
\end{tabular}




\begin{tabular}{|l|c|}
\hline Por propiedad conmutativa & $x=3-2 m$ \\
\hline Sustituyendo las variables originales & $\frac{s}{s_{o}}=3-2 \frac{\alpha S_{o}}{n \delta}$ \\
\hline
\end{tabular}

Fuente: Elaboración propia.

Con la expresión anterior se puede estimar a qué nivel la población ha bajado en el brote epidémico, teniendo presente que $\frac{\alpha S_{o}}{n \delta}>1$, si este valor es mayor que 1 al multiplicarlo por 2 se obtendrá un valor mayor a dos, y al restarlo por tres se tendrá una fracción que se podrá relacionar con $\frac{s}{s_{o}}$ sabiendo que $s$ va ser un poco menor a $s_{o}$. De esta manera podrá calcular la población que resta de susceptibles que fueron infectados, como también las personas que se recuperaron. $R=n-S$.

$$
\frac{R}{S_{o}}=\frac{n-S}{S_{o}}=\frac{n}{S_{o}}-3+2 \frac{\alpha S_{o}}{n \delta}
$$

Se puede tener una idea de la cantidad de personas afectadas por una estimación basado en $S \sim S_{o}$ teniendo un máximo un 1, 5 inclusive hasta un $10 \%$ de la población, si fuera más se tendrá que entrar a órdenes superiores. Con el argumento que se expuso anteriormente, lo que se busca es que $R_{o}<1$ para tener control y poder salir de la pandemia. Aunque este no es el caso de Latinoamérica pues la pandemia sigue latente por lo tanto $R_{o}>1$. En china se logró llegar a este valor con muchos cuidados.

El gobierno chino ha usado herramientas tecnológicas innovadoras y polémicas, como el rastreo de teléfonos. Y otros países empiezan a mirar a soluciones similares. Hay robots repartiendo la comida en los hospitales, cámaras de reconocimiento facial que controlan la temperatura de las personas, y hasta drones que vigilan que la gente cumpla con la cuarentena. Sin embargo, el sistema chino de control de masas ha sido criticado de forma constante por diversos grupos de derechos humanos y digitales en diferentes países (Bbc, 2020, p. 1).

En Guatemala se carece de tecnología que permita igualar protocolos similares a los de países como China, no obstante, se tendría que seguir de manera diligente las recomendaciones de las autoridades gubernamentales, las cuales son publicadas en los diferentes medios de comunicación del país y redes sociales, con el fin de evitar el aumento de casos confirmados. 
Yojcom, D. \& Ruiz, J. (2020). Los factores sociales asociados a los modelos matemáticos en un contexto de pandemia. Revista Latinoamericana de Etnomatemática, 13(1), 355-374. DOI: 10.22267/relatem.20131.47

Aunque, el parámetro de contagio $\alpha c$ puede ser normalizado con el uso adecuado de mascarillas y la permanencia en cuarentena, evitando así la actividad de contactos $c$. Según la OMS, señala la diferencia entre brote, epidemia y pandemia.

Un brote epidémico es una clasificación usada en la epidemiología para denominar la aparición repentina de una enfermedad debida a una infección en un lugar específico y en un momento determinado. Por su parte, se cataloga como epidemia cuando una enfermedad se propaga activamente debido a que el brote se descontrola y se mantiene en el tiempo. De esta forma, aumenta el número de casos en un área geográfica concreta. Para que se declare el estado de pandemia se tienen que cumplir dos criterios: que el brote epidémico afecte a más de un continente y que los casos de cada país ya no sean importados sino provocados por trasmisión comunitaria (Pulido, 2020, párrafo 4).

En el caso de COVID-19 se declaró como pandemia, siendo $R_{o}>1$, afectando a los demás continentes por los criterios que señala la OMS. Lamentablemente no se ha encontrado una vacuna o medicamento que prevenga y erradique este virus, de aquí la importancia de los factores contextuales para obtener $R_{o}<1$ con el uso de mascarillas y permanencia en cuarentena. Correlacionando del parámetro $\alpha$ con el uso de mascarillas y $c$ con el evitar contactos.

Con este modelo se puede complementar con la premisa "que la matemática es una construcción social". En la actualidad el modelo teórico SIR ayuda estimar y pensar en acciones que se pueden viabilizar, buscando el cuidado colectivo y el bienestar social. El avance de las ciencias nos permitirá tener mejores panoramas, cambiando las cifras de los afectados y logrando responder positivamente ante la incertidumbre.

Es importante señalar que el virus es adquirido de diversas maneras, Ravin (2020) indica cómo una persona puede ser contagiada con un esturnudo, esparciendo pequeñas gotas de líquido en el aire, las cuales pueden llegar a la nariz, la boca o los ojos de alguien cercano. El estornudo tiene una velocidad promedio de 110 a $170 \mathrm{~km} / \mathrm{h}$ (Manzanas, 2019); aunque la OMS señala que el coronavirus puede expandirse hasta una distancia de 8 metros a través del estornudo de una persona enferma con Covid-19 (Redacción médica, 2020).

...el patógeno es capaz de aguantar tres horas de media tras haber sido expulsado en forma pequeñas gotitas a través de la tos o estornudos. La OMS recuerda que ese estudio se ha llevado a cabo en el laboratorio, usando aparatos nebulizadores que no reflejan las condiciones reales de la tos o el estornudo humanos (Sáens, 2020, p. 1). 
Con los datos señalados, se puede comprender la importancia del distanciamiento social y de otros factores, pues una persona al estornudar, expulsa partículas por medio de la saliva, las cuales pueden durar hasta por tres horas.

\section{IMPORTANCIA DEL ANÁLISIS DE LOS FACTORES ASOCIADOS A APLICACIÓN DE MODELOS MATEMÁTICOS EN LA ESTIMACIÓN DE FENÓMENOS SOCIALES Y CULTURALES.}

Las informaciones obtenidas de los modelos matemáticos ofrecen una mirada de los

fenómenos. Sin embargo, los entes biológicos y sociales están condicionados fuertemente por el contexto, porque los seres vivos no poseen patrones regulares que puedan modelar su comportamiento. Así mismo, existen patrones culturales y sociales que hacen que tengan un mayor o menor número de contagio, según sea el caso. De esta manera el contexto o el medio juegan un rol importante en el desarrollo de los seres vivos.

Bajo esta mirada Huang (2008), sugiere una nueva imagen de la racionalidad para interpretar y comprender los fenómenos, porque a consideración de él, la fuerza normativa de los principios del razonamiento tiene que ver con los factores contextuales de sus aplicaciones. Por lo que propone una función básica de interpretar los fenómenos en un ambiente natural dado por $y=\mathrm{F}(\mathrm{x}, \mathrm{k})$, en donde $y$ es la información inferida, $F$ el proceso computacional de inferencia, $x$ la información original y $k$ es una serie de factores contextuales. De aquí surge la importancia de nuestra reflexión, los factores contextuales son diversos y poseen múltiples formas de poder interpretarlos.

Dentro de las investigaciones que se inscriben en el marco de la etnomatemática, no es posible ignorar la importancia de los factores contextuales en nuestra reflexión socioeducativa, porque es innegable el valor del contexto, por lo que el concepto de racionalidad contextual utilizada por Huang cobra importancia en este paradigma, ¿En qué se funda la racionalidad propuesta por Xiang Huang?

La racionalidad no reside únicamente en pensar o actuar de acuerdo con un conjunto de reglas de razonamiento sino también en examinar si la aplicación de una regla es adecuada o no dentro del contexto de una práctica específica, y que, por otro lado, diferentes principios normativos del razonamiento requieren que los criterios de corrección de sus aplicaciones se especifiquen mediante una serie de factores 
contextuales específicos que configuran diferentes tipos de contexto (Huang, 2008, p. $15)$.

Las reglas utilizadas para estimar según el modelo SIR están fuertemente discutidas en los párrafos anteriores, ahora corresponde analizar los factores asociados a la aplicación del modelo SIR dentro de un contexto guatemalteco. Nuestra intención no es enumerar todos los factores que pueden incidir en la aplicación de estos conocimientos matemáticos, sino mencionar aquellos que según nuestra perspectiva tienen más impacto en la sociedad:

Capacidad de relacionar y asociar datos matemáticos. La escuela tradicional, se ha esforzado por enseñar a leer, escribir y realizar operaciones básicas aritméticas. Muchos de estos "contenidos" han sido descontextualizados, recurriendo solamente a la aplicación de reglas aprendidas a través de ejercicios repetitivos, dejando a un lado la resolución de problemas de la vida cotidiana. Rara vez observamos el uso de la información estadística que reportan los periódicos, para el desarrollo de una clase de matemáticas, difícilmente un estudiante del nivel medio pueda justificar si la cotización del precio del azúcar es más barata en Nueva York o en Guatemala, lo que diariamente la prensa local informa a la población sobre este comportamiento económico. Caso muy parecido sucedió con los datos y los pronósticos sobre los picos altos que se podían dar sobre el comportamiento del coronavirus en el país, simplemente no hubo una "buena lectura” de esta información. Los conocimientos matemáticos de la comunidad académica fueron fuertemente conocidos y difundidos, sin embargo, la lectura y la interpretación dada por las personas, las organizaciones y autoridades en turno, no fueron las más acertadas ni las más apegadas a estos modelos, lo cual es una clara evidencia de la poca capacidad de asociar datos inferidos a contextos locales.

Vinculación de resultados al conocimiento unitario (Bachelard, 2000), menciona que se debe apostar no sólo por la diversidad del conocimiento humano, sino por la diversidad de procesos que dan sentido a esos conocimientos. La generalización es importante para comprender el comportamiento del fenómeno, pero el desconocimiento de los elementos y factores implícitos no pueden estar al margen del análisis, por eso "la necesidad de generalizar hasta el extremo, a veces mediante un solo concepto, arrastra a ciertas ideas sintéticas que están lejos de perder su poder de seducción” (Bachelard, 2000, p. 113). Para el caso guatemalteco, no sólo no se pudo dar una "buena lectura" a los datos, sino se comenzó a implementar acciones impulsadas por otros países, aún sin conocer el comportamiento o el 
ciclo biológico del virus. Es evidente que en pleno siglo XXI el conocimiento unitario y pragmático sigue siendo un obstáculo para el desarrollo y sobre todo para la investigación y promoción de la ciencia, desde una diversidad epistemológica.

La cultura como motor de desarrollo local, las dinámicas poblacionales afectan directa o indirectamente el comportamiento de los organismos vivos que se encuentran en el entorno, de igual forma, se considera que ha habido una discriminación del conocimiento ancestral, pues los jefes de Estado se apegaron a los protocolos emitidos por la OMS, ignorando las prácticas ancestrales y la medicina alterna que podía combatir este virus. La interacción continua entre los seres humanos y las prácticas culturales determinan en un buen porcentaje la propagación y el control de la reproducción de distintas enfermedades. Uno de los detonantes fuertes de esta enfermedad es la falta de higiene y limpieza. No todas las familias poseen los mismos hábitos de limpieza, en definitiva, estos hábitos están vinculados con la educación y la cultura. UNICEF (2020) menciona que todo es importante desde la higiene personal, limpieza del hogar, lavado de ropa, manipulación y preparación de los alimentos para contrarrestar el COVID-19.

La naturaleza del fenómeno de estudio. Como se ha dicho anteriormente la biología del coronavirus es poco conocida en el mundo, no existe un historial que pueda utilizarse para predecir con mayor precisión su comportamiento. Los fenómenos estudiados por el hombre como la electricidad y el magnetismo han trazado su propia historia, con el pasar de los años estos descubrimientos fueron más accesibles para la humanidad al igual que su utilidad en ciertos ámbitos de la vida.

Se puede evidenciar la importancia de la matemática, pues aporta conocimientos para el desarrollo de la tecnología y cuidados de salud, por consecuente es necesario el crecimiento científico en nuestro país, así como profesionales que puedan compartir este conocimiento de manera eficiente a las nuevas generaciones, con el fin de lograr mejores aportes de ciencias aplicadas (tecnologías), y así poder buscar soluciones viables en los tiempos de contingencia como el que se está viviendo.

La evolución como uno de los principios básicos de la vida. La noción de evolución en este texto puede entenderse desde dos perspectivas: a) Desde la visión darwiniana, que establece que todos los seres vivos evolucionan y hay una constante lucha por la 
supervivencia, en este proceso de competencia sobrevive el más apto. b) Desde la visión autopoiética, entendida como aquel microorganismo que "describe un fenómeno radicalmente circular: las moléculas orgánicas formas redes de reacciones que producen a las mismas moléculas de las que están integradas (Maturana, 2009, p. XIII). Con base a esta última perspectiva, se comprende que los nichos ecológicos son cambiantes y los organismos no se adaptan ajenamente a éstos, sino evolucionan con ellos. Entonces, para el modelo matemático que pretende estimar el comportamiento del COVID-19, no puede ignorar la lucha por la supervivencia del más apto ni la evolución de los nichos ecológicos que indiscutiblemente son factores intrínsecos a este modelo.

La educación, una de las opciones poco reconocidas para la prevención y control de enfermedades en Guatemala. La educación es un salvavidas para los niños y jóvenes, porque la escuela puede ser una fuente valiosa de aprendizajes sobre salud y prevención de riesgos, y en colaboración con los padres de familia, puede ayudar a canalizar las emociones de aquellos niños y jóvenes que se han visto expuestos a informaciones inapropiadas para su edad (UNICEF, 2020). La educación escolarizada como la educación no escolarizada influyen significativamente en la buena salud de los habitantes. Educar a los niños y jóvenes a asumir responsabilidades, a garantizar su integridad física, a llevar un control periódico y sistemático de su estado de salud, son prácticas que deben ser fortalecidos en los diferentes centros educativos. La escuela es una de las instituciones de referencia en muchas comunidades del país, y por lo tanto, la información y la educación que pueda salir de estos espacios educativos tiene grandes implicaciones en la vida cotidiana de la población.

Capacidad de reacción para atender emergencia, todo ser humano esta propenso de experimentar algún tipo de emergencia, especialmente las relacionadas a salud que han azotado a diferentes culturas, como en los casos de: peste negra, viruela, gripe española, entre otros. La historia reporta cifras alarmantes de muertes provocadas por estas pandemias, sin embargo, en el contexto nacional no se tiene un plan de prevención de Estado que pueda atender estas emergencias ni esfuerzo conjuntos que permita articular las prácticas ancestrales y la medicina moderna. Por otro lado, la capacidad instalada y la cantidad de médicos en servicio no son suficientes para atender casos masivos en el país. 


\section{CONSIDERACIONES FINALES}

La historia es un elemento importante para analizar longitudinalmente los comportamientos y casos que ha experimentado la humanidad. Estos datos, sucesos y hechos que son reportados por la historia deben ser considerados para proponer y diseñar nuevas estrategias que permitan conservar y prevenir la buena salud de los habitantes del planeta.

Los modelos matemáticos son herramientas fundamentales para generalizar comportamientos y fenómenos entre dos o más variables para comprender la naturaleza, y su análisis no es un acto antojadizo ni caprichoso; sino debe realizarse bajo reglas y criterios técnicos que permitan estimar resultados lo más ajustado posible a la realidad.

Los factores asociados a los modelos matemáticos condicionan la adecuada aplicación, interpretación e institucionalización de resultados en diferentes contextos. La diversidad cultural y las diferentes cosmovisiones e interpretaciones endógenas de los grupos sociales deben ser valorados e incorporados en la resolución de problemas locales, nacionales e internacionales.

Las repercusiones que posee la historia, los modelos matemáticos y los factores asociados en la generalización de esos constructos, permite valorar los conocimientos locales y la sabiduría ancestral, para hacer estimaciones legítimas que garantice la apropiación de los procesos y la provocación de una consciencia humana que persiga el bien común. 


\section{REFERENCIAS}

Bachelard, G. (2000). La Formación del Espíritu Científico. México: Siglo XXI Editores.

Bbc (2020). Coronavirus en China: Cómo funciona el sofisticado y polémico sistema de vigilancia para controlar la pandemia. Recuperado de https://www.bbc.com/mundo/noticias-52361034

France24 (2020). El exitoso método de Alemania para lograr menos muertes por coronavirus. Recopilado de: https://www.france24.com/es/20200330-alemaniametodo-exitoso-pruebas-aparato-salud-covid19

Cervantes, L. (2015). Modelización matemática principios y aplicaciones. Puebla, México: Benemérita Universidad Autónoma de Puebla.

Ecured (2020). Matemática aplicada. Recopilado de https://www.ecured.cu/Matem\%C3\%A1tica_aplicada

Fernández, V. (2018). Modelos Mecanísticos Determinísticos con Ecuaciones Diferenciales. Recopilado de https://rpubs.com/dsfernandez/422937

Galindo, S., Rodríguez, M. \& Cervantes, J. (2014). Las matemáticas de las epidemias: Caso México 2009 y otros. Ciencia ergo-sum, 20(3), 238-246.

Huang, X. (2008). De la Racionalidad Tradicional a la Racionalidad Contextual. México: Publicaciones Cruz O., S. A.

Gerber, W. (2012). Epidemiología Modelo SIR. Chile. Recopilado de https://www.youtube.com/watch?v=-2101hLmaDw .

Girón, M. (2020). Según Salud se han hecho más de 12 mil prueba de COVID-19. La Hora. Recopilado de https://lahora.gt/segun-salud-se-han-hecho-mas-de-12-mil-pruebasde-covid-19/

Gramajo, J. (2020). Covid-19: esta es la cantidad de pruebas que tiene el Gobierno. Soy 502. Recopilado de https://www.soy502.com/articulo/esta-cantidad-pruebas-tiene-covid19-gobierno-100931

Herrero, M. (2017). Así ayudaron las matemáticas a calcular la propagación de epidemias. El país. Recopilado de https://elpais.com/elpais/2017/02/06/ciencia/1486386507_636571.html

Kermack, W. \& McKendrick (1927). Contribution to the Mathematical Theory of Epidemics. Recopilado de https://royalsocietypublishing.org/doi/10.1098/rspa.1927.0118

Manzanas, J. (2019). ¿A qué velocidad viaja un estornudo?. okdiario. Recopilado de https://okdiario.com/curiosidades/que-velocidad-viaja-estornudo-4825984

Maturana, H. (2009). La Realidad: ¿Objetiva o Construida? I Fundamentos Biológicos de la Realidad. España: Anthropos. 
Pulido, S. (2020). ¿Cuál es la diferencia entre brote, epidemia y pandemia. Gaceta Medica. Recopilado de: https://gacetamedica.com/investigacion/cual-es-la-diferencia-entrebrote-epidemia-y-pandemia/

Ravin, K. (2020). Coronavirus (COVID-19). kidshealth. Recopilado de https://kidshealth.org/es/parents/coronavirus-esp.html

Redacción médica (2020). Cómo se contagia el coronavirus: estornudos con hasta 8 metros de alcance. Redaccionmedica. Recopilado de https://www.redaccionmedica.com/secciones/sanidad-hoy/coronavirus-como-secontagia-estornudos-8-metros-de-alcance-1317

Roldán, P. (2020). Ley de los grandes números. Economipedia. Obtenido de https://economipedia.com/definiciones/ley-los-grandes-numeros.html.

Sáens, C. (2020). El coronavirus no se transmite a distancia por el aire advierte la OMS. Lavanguardia.

Obtenido

de https://www.lavanguardia.com/vida/20200329/48147148995/coronavirus-covid-19oms-aire-transmision-contagio.html

UNICEF (2020). El nuevo coronavirus y el derecho a la educación. Obtenido de https://www.unicef.es/educa/blog/nuevo-coronavirus-derecho-educacion 